\title{
La enseñanza de la estadística y de las técnicas de investigación social a distancia
}

\author{
luis A. Camarero, \\ Marisa García de Cortázar \\ Y CONSUELO DEL VAL \\ Departamento de Sociología I (UNED)
}

1 $\mathrm{N}$ el año académico 1987-88 se puso en marcha el primer curso de las licenciaturas de Ciencias Políticas y Sociología en la Universidad Nacional de Educación a Distancia. Hasta entonces la única Universidad Pública que impartía estas carreras era la Complutense de Madrid. Esto significaba que a pesar del interés por la Ciencia Política y la Sociología existente en el país, tan sólo los residentes de la Comunidad de Madrid o los que a ella se trasladaran, podían cursar estos estudios oficialmente.

Esta situación propició el enorme éxito de matrícula con que contó la nueva Facultad desde sus inicios (más de 2.000 alumnos), ya que rompía con la limitación geográfica anterior. Cualquier alumno desde cualquier punto de España podía seguir estos estudios.

Años más tarde la Facultad ha mantenido una tendencia ininterrumpida de crecimiento (en torno a los ocho mil alumnos) y cuenta ya con varias promociones de licenciados. Desde esta perspectiva temporal, consideramos de interés sondear a los licenciados de las tres primeras promociones (alrededor de 250) acerca de su experiencia como alumnos de la Facultad y, específicamente, como alumnos de las materias metodologicas comunes a ambas carreras. Para ello se elaboró un cuestionario que fue enviado por correo a la totalidad de licenciados a las direcciones que figuraban en sus respectivos expedientes. En muchos casos esta dirección no correspondía con la más actual, como demostró la tasa de devolución, cercana al treinta por ciento. Si bien este porcentaje puede calificarse de discreto, se comprobó la existencia de una correspondencia proporcional entre el número de los graduados existentes y los licenciados que han respondido de las diferentes promociones.

El número de cuestionarios válidos nos llevó a efectuar un análisis básico de los datos de la encuesta, cuya interpretación desarrollamos a continuación. 


\section{1. ¿QUIÉNES SON LOS LICENCIADOS?}

En estas tres primeras promociones los licenciados en Sociología sobrepasan a los de Ciencias Políticas (56,7\% frente a 43,3\%). Esta diferencia responde a la distribución de alumnos matriculados en la Facultad en las dos secciones en los primeros años. Desde 1992, los alumnos de Ciencias Políticas no sólo se han igualado a los de Sociología, sino que los han sobrepasado con creces.

Centrándonos más en el perfil de los primeros licenciados de la Facultad, llama la atención el fuerte predominio de los varones, ya que dos de cada tres licenciados son del sexo masculino. Si se establece la comparación por carreras, el caso de Ciencias Políticas agudiza el desequilibrio, puesto que sólo un $20 \%$ de los licenciados en esta carrera es mujer.

Además de esta asimetría de género, en una institución como la Universidad española en la que la proporción de graduadas es, incluso, superior a la de graduados, sorprende la edad de nuestros licenciados. La mitad de ellos tiene en la actualidad 35 años o más, lo que implica que la práctica totalidad de ellos comenzaron sus estudios de Sociología o CC. Políticas a la edad en que sus compañeros de otras universidades concluyeron los suyos. Estudiar Sociología o CC. Políticas ha supuesto para ellos una elección meditada.

TABLA 1

Edad de los licenciados

\begin{tabular}{lcc}
\hline EDAD & FRECUENCIA & PORCENTAJE \\
\hline$>30$ & 13 & 19,7 \\
$30-35$ & 19 & 28,85 \\
$36-40$ & 14 & 21,2 \\
$41-45$ & 15 & 22,7 \\
$>46$ & 5 & 7,6 \\
\hline TOTAL & 66 & 100,0 \\
\hline
\end{tabular}

Esta hipótesis se ve reforzada al examinar los antecedentes educativos de nuestros licenciados. Dos de cada tres acceden a los estudios de la Facultad después de haber cursado parte de otros estudios superiores $(31,3 \%)$ o, incluso, tras estar en posesión de otro título universitario $(34,3 \%)$. Es decir, menos de un tercio comienzan sus estudios sin experiencia universitaria previa, la situación de partida habitual para otros estudiantes.

También difieren del resto de sus compañeros de la Universidad en aspectos tales como el trabajo y las responsabilidades familiares. Nuestros licenciados, cuando inician los estudios desempeñan un trabajo en tres de cada cuatro casos y sólo una exigua minoría $(15,4 \%)$ dedica todo su tiempo al estudio. Por otra parte, más de la mitad de ellos estaban casados y con hijos. Trabajo y familia, dos circunstancias que hubieran limitado las posibilidades de continuar estudios en una universidad tradicional, dejan de ser impedimentos al disponer de una alternativa como la ofrecida por la UNED. 
TABLA 2

Formación al iniciar los estudios en la UNED

\begin{tabular}{lcc}
\hline ESTUDIOS DE ACCESO & FRECUENCIA & PORCENTAJE \\
\hline Selectividad & 18 & 26,9 \\
Acceso mayores 25 & 5 & 7,5 \\
Otros super comenz. & 21 & 31,3 \\
Título licenciado & 23 & 34,3 \\
\hline TOTAL & 67 & 100,0 \\
\hline
\end{tabular}

TABLA 3

Situación profesional al comenzar los estudios en la UNED

\begin{tabular}{lcc}
\hline SITUACIÓN PROFESIONAL & FRECUENCIA & PORCENTAJE \\
CUANDO ESTUDIABAN & 3 & 4,6 \\
\hline Trab. por cuenta propia & 44 & 67,7 \\
Trab. por cuenta ajena & 3 & 4,6 \\
Negocio familiar & 3 & 4,6 \\
Ama de casa & 10 & 15,4 \\
Solo estudia & 1 & 1,5 \\
Parado & 1 & 1,5 \\
Servicio Militar & 65 & 100,0 \\
\hline TOTAL & & \\
\hline
\end{tabular}

Las distancias geográficas también se ven atenuadas. El lugar de residencia no condiciona la posibilidad de estudiar. De hecho, el $40 \%$ de los licenciados ha podido graduarse residiendo en ciudades de menos de cien mil habitantes, lugares en los que normalmente no han estado radicadas facultades universitarias.

\section{COMPORTAMIENTO ACADÉMICO}

Todo lo anteriormente expuesto refleja la peculiaridad de nuestros licenciados si nos atenemos a sus características personales. También es singular la forma en que han cursado la carrera y cómo han enfocado el estudio de las materias de Estadística y Técnicas de Investigación.

El mayor número de licenciados que han contestado a la encuesta $(40,3 \%)$ iniciaron sus estudios el primer año de funcionamiento de la Facultad, en el curso 1987/88. De haber mantenido un ritmo de progresión regular en sus estudios, cinco años después deberían figurar como licenciados. Sin embargo, la proporción de licenciados que pertenece a la primera promoción representa el $28 \%$. Similar adelgazamiento se produce en la promoción siguiente, 
mientras que la última, la del 93/94, recoge la mitad de los licenciados. La primera conclusión es que estos primeros licenciados emplean más de los cinco años reglamentarios en cursar sus estudios, retraso explicable por sus especiales circunstancias personales y quizá, también, por las inevitables dificultades y carencias organizativas de una Facultad novicia.

TABLA 4

Años de comienzo y término de la carrera en la UNED

\begin{tabular}{lcc}
\hline $\begin{array}{l}\text { AÑO DE COMIENZO } \\
\text { CARRERA }\end{array}$ & FRECUENCIA & PORCENTAJE \\
\hline $87-88$ & 27 & 40,3 \\
$88-89$ & 19 & 28,4 \\
$89-90$ & 15 & 22,4 \\
$90-91$ & 5 & 7,5 \\
$91-92$ & 1 & 1,5 \\
\hline TOTAL & 67 & 100,0 \\
\hline AÑO TÉRMINO & FRECUENCIA & PORCENTAJE \\
CARRERA & 19 & 28,4 \\
\hline $91-92$ & 15 & 22,4 \\
92-93 & 33 & 49,3 \\
93-94 & 67 & 100,0 \\
\hline TOTAL & & \\
\hline
\end{tabular}

En este retraso, la asignatura de Estadística ha tenido alguna responsabilidad. Los licenciados han diferido en muchos casos la matrícula en esta asignatura, cursándola, no en el primer año como está establecido en el curriculum, sino en años posteriores. Así, mientras el $68,7 \%$ de los respondientes se había matriculado en la Facultad en los dos primeros años de funcionamiento, tan s6́lo aparece el 34,4\% matriculado en Estadística en ese período. La imagen estereotipada de asignatura «matemática» y difícil ha podido influir en esta pauta de conducta, que sin embargo no se da en el caso de Técnicas de Investigación, materia en la que se mantiene una relación de matrícula y curso mucho más proporcional.

Los licenciados bajo estudio, una vez matriculados en Estadística no tuvieron muchas dificultades en aprobar y la mayoría lo hizo en el curso de la primera matrícula, aunque los alumnos del primer año de funcionamiento encontraron mayores problemas en lograr su objetivo. Lo mismo sucedió con la asignatura de Técnicas de Investigación; casi todos los licenciados superaron la materia en el tiempo establecido.

Además de conseguir pasar estas materias en el tiempo previsto, las calificaciones que obtuvieron pueden calificarse de «buenas», dado el tradicional rigor evaluativo de la UNED. En el caso de Estadística, hay el mismo por- 
centaje de licenciados con la papeleta de «Aprobado» (41\%), que con la de «Notable», e incluso el $10 \%$ ha sido merecedor de una nota de «Sobresaliente». En Técnicas, la dispersión de calificaciones es menor, siendo la más habitual la de «Aprobado» $(67,7 \%)$. Las calificaciones son consideradas satisfactorias por la mayoría de los alumnos. A la pregunta de si la nota obtenida en estas materias se ajustaba a sus expectativas, tres de cada cuatro responden que «mucho» 0 «bastante» al referirse a Estadística, porcentaje que desciende al 58\% en el caso de Técnicas de Investigación Social.

\section{ESTUDIANTES SOLITARIOS}

En función de lo anteriormente expuesto puede afirmarse que estos primeros licenciados no se tropezaron con obstáculos extraordinarios para cursar estas materias, pese a tratarse de asignaturas que necesitan de una docencia más práctica y personal que otras disciplinas de las carreras de Sociología y Ciencias Políticas. Por ello, es interesante conocer cómo se enfrentaron a su estudio y en qué grado hicieron uso de los recursos específicos que la UNED tiene establecidos para ayudar al estudiante en su labor.

Casi el 80 por ciento de los licenciados que han contestado a la encuesta, contaban con tutores para estas materias en los Centros Asociados en que se impartía la carrera. Y sin embargo, no han utilizado este recurso con la intensidad prevista. Teniendo en cuenta que la tutoría de Estadística y Técnicas de Investigación se imparte una vez a la semana, sólo el $29,8 \%$ de los licenciados acudieron «casi siempre» a la tutoría de la primera y menos de un $15 \%$ lo hicieron a la de la segunda. Una posible explicación a tan discreta frecuentación puede encontrarse en la distancia que debían recorrer para acudir a las mismas pues la mayoría de los licenciados no tenían un centro asociado en su localidad de residencia, teniendo que desplazarse cada vez que acudían al mismo. Es claro que esta circunstancia ha podido frenar la asistencia a tutorias, pero no es la única.

TABLA 5

Asistencia a tutorías

\begin{tabular}{lrrlrr}
\hline $\begin{array}{l}\text { Asistencia } \\
\text { tutorías } \\
\text { Estadistica }\end{array}$ & Frecuencia & Porcentaje & $\begin{array}{c}\text { Asistencia } \\
\text { tutoría } \\
\text { Técnicas }\end{array}$ & Frecuencia & Porcentaje \\
\hline Casi siempre & 17 & 29,8 & Casi siempre & 9 & 14,5 \\
Frecuent. & 4 & 7,0 & Frecuent. & 3 & 4,8 \\
De vez en c. & 9 & 15,8 & De vez en c. & 12 & 19,4 \\
Nunca & 18 & 31,6 & Nunca & 21 & 33,9 \\
No había & 9 & 15,8 & No había & 17 & 27,4 \\
\hline TOTAL & 57 & 100,0 & TOTAL & 62 & 100,0 \\
\hline
\end{tabular}


El examen del uso de otros recursos educativos de la UNED que no precisan de ningún desplazamiento, hace pensar que estos licenciados se plantearon el estudio de estas materias de forma «autodidacta», estudiando los libros recomendados en solitario y sin recurrir a ayudas exteriores. Quizá, porque a su juicio (el $75 \%$ de las respuestas), los textos eran suficientes para la preparación de estas materias. Así, cuando se les pregunta por el número de consultas que realizaron a los profesores de la sede central, las respuestas fueron bastante desalentadoras: la mitad de los licenciados nunca formularon ninguna consulta telefónica sobre la asignaturas de Estadística y Técnicas de Investigación, pese a existir un servicio de guardias del profesorado creado para este propósito; cerca del $80 \%$ jamás se entrevistó con los profesores y cerca del $90 \%$ no formuló consultas postales. Es decir, los licenciados no utilizaron los servicios del profesorado de estas asignaturas, por ninguno de los medios previstos. Tampoco escucharon las emisiones de radio dedicadas a estas materias y no pudieron asistir a convivencias (encuentros entre el profesorado y los centros asociados), porque en estos primeros años apenas se realizaron. Estudiaron valiéndose de sus propios recursos y no considerando las ayudas que hubieran podido recibir de profesores y tutores. Ni siquiera la de sus propios compañeros, porque como declaran los entrevistados, la forma de estudio habitual era en soledad.

\section{LA EVALUACIÓN DE LAS ASIGNATURAS DE METODOLOGÍA (No es tan fiero el lobo como lo pintan)}

Antes de acercarnos a las opiniones que les merecieron las asignaturas de metodología a los licenciados en CC. Políticas y Sociología de la UNED, no estaría de más tener en cuenta que la fragilidad de la memoria inherente al paso del tiempo ha condicionado considerablemente las informaciones proporcionadas por los encuestados. Debemos considerar que las asignaturas de «Estadística» y «Técnicas de Investigación Social» se enmarcan en los dos primeros cursos de ambas licenciaturas, por lo que no ha de resultar fácil para los informantes reconstruir con nitidez las expectativas, impresiones y opiniones que al respecto de las mismas podrían tener hace cinco años o más. Debido a este problema, aproximadamente un tercio del total de los licenciados no ha contestado a preguntas como las dirigidas a la obtención de información sobre exámenes, carencias y comparación de los contenidos de las asignaturas impartidas en otras Universidades. Hecha esta salvedad, pasaremos a examinar la perspectiva ofrecida por nuestros licenciados.

Se podría comenzar por decir que su experiencia con las asignaturas de metodología ha sido, en líneas generales, satisfactoria. Así, si nos centramos en la percepción que tienen de aquellos aspectos que podrían haber influido más decisivamente en el resultado académico (calificaciones), tales como los relacionados directamente con la adecuación de los exámenes, se observa que «evalúan a los evaluadores» de un modo que podríamos calificar de generoso. Los exámenes de Estadística y de Técnicas son recordados por más del $80 \%$ de los que respondieron como no excesivamente díficiles, sin visos de 
inducir a equivocaciones y con una clara correspondencia con los textos recomendados. No obstante, se aprecian algunas diferencias entre la imagen dada por las dos asignaturas en lo que respecta a la estructuración y a la previsibilidad de las mismas, siendo mayor el volumen de los que consideran las de Estadística como más estructuradas y previsibles que las de Técnicas, aspecto que concuerda claramente con el mayor peso práctico que caracteriza tradicionalmente a los exámenes de aquella asignatura frente a los de ésta.

Esta evaluación positiva de los aspectos más concretos de la enseñanza se refuerza cuando pasamos a una esfera más ideal o abstracta como la siempre traída y llevada adecuación de los contenidos impartidos en la Universidad a las necesidades del mundo del mercado de trabajo. Un 72,7\% de los que respondieron considera que el contenido de la asignatura de Estadística se adecúa mucho o bastante a las necesidades profesionales de un sociológo o politólogo, en tanto que adoptan una postura más crítica con los contenidos de Técnicas, reduciéndose este porcentaje a un $54,8 \%$.

TABLA 6

Percepción de la adecuación profesional de los contenidos de las asignaturas de estadística y técnicas de investigación social

\begin{tabular}{lcc}
\hline ADECUACIÓN PROFESIÓN & FRECUENCIA & PORCENTAJE \\
\hline CONTENIDO ESTADISTICA & 8 & 14,5 \\
Mucho & 32 & 58,2 \\
Bastante & 13 & 23,6 \\
Poco & 2 & 3,6 \\
Nada & 55 & 100,0 \\
\hline TOTAL & FRECUENCIA & PORCENTAJE \\
\hline ADECUACIÓN PROFESIÓN & 10 & 16,1 \\
CONTENIDO TÉCNICAS & 24 & 38,7 \\
\hline Mucho & 24 & 38,7 \\
Bastante & 4 & 6,5 \\
Poco & 62 & 100,0 \\
Nada & & \\
\hline TOTAL & & \\
\hline
\end{tabular}

Aun así, no se resisten a reflejar las principales carencias que achacan a la especificidad de estas asignaturas en el ya peculiar marco de la enseñanza a distancia. La demanda por excelencia es la de la necesidad de ampliar la dimensión práctica de ambas asignaturas, agudizándose en el caso de las Técnicas, mientras en Estadística también se solicita el perfeccionamiento de los materiales didácticos y, fundamentalmente, el mayor uso de medios audiovisuales. Esta petición es, si cabe, más característica en el ámbito de la UNED, donde se hace especial hincapié en estos aspectos. 
Para finalizar con la evaluación de los contenidos de estas materias pasaremos a la comparación que establecen con los programas impartidos en otras Universidades y de los que tienen conocimiento a través de los contactos que establecen con sus alumnos. Partiendo de que sólo un tercio de nuestros licenciados afirma tener conocidos en otras Universidades, tres de cada cuatro de ellos responden que la asignatura de Estadística es más completa en la UNED, en tanto que más de la mitad confirman su mayor dificultad.

Tras hacer esta comparación entre universidades, sólo queda el contraste interno, en el contexto del resto de las asignaturas de la carrera. En una escala del 1 al 7, si bien las Técnicas de Investigación se ven mayoritariamente ubicadas en el punto central (ni más fácil ni más difícil que las demás), la distribución resultante respecto a Estadística tiende a sesgarse hacia los valores más altos, los de más dificultad.

Por tanto la clásica dureza atribuida a las asignaturas de componente empírico en las carreras de Humanidades no desaparece en el caso que nos ocupa.

\section{TABLA 7}

Percepción de la dificultad de las asignaturas de Estadística y de Técnicas de investigación social

\begin{tabular}{cccccr}
\hline $\begin{array}{c}\text { Dificultad } \\
\text { relativa } \\
\text { estadística }\end{array}$ & Frecuencia & Porcentaje & $\begin{array}{c}\text { Dificultad } \\
\text { relativa } \\
\text { técnica }\end{array}$ & Frecuencia & Porcentaje \\
\hline 1 & 1 & 1,7 & 1 & 2 & 3,1 \\
$2-3$ & 9 & 15,5 & $2-3$ & 13 & 20,3 \\
4 & 16 & 27,6 & 4 & 29 & 45,3 \\
$5-6$ & 25 & 43,1 & $5-6$ & 15 & 23,5 \\
7 & 7 & 12,1 & 7 & 5 & 7,8 \\
\hline TOTAL & 58 & 100,0 & TOTAL & 64 & 100,0 \\
\hline
\end{tabular}

A pesar de la dificultad atribuida a estas materias se observa entre nuestros licenciados un gran interés, como hemos visto más arriba, por su componente práctico, algo que concuerda con la percepción que tienen de la importancia de las mismas en el ámbito profesional. Del total de cuestionarios recibidos el $78 \%$ presenta la Estadística como una materia imprescindible o muy importante de cara a la práctica profesional de un sociólogo/politólogo, acentuándose en el caso de las Técnicas, que es considerada del mismo modo por un $84,4 \%$ de los mismos.

Claro está que, a posteriori, tras licenciarse, la percepción de la relevancia de las asignaturas de carácter metodológico puede verse modificada, pero en general, si hacemos una comparación entre el recuerdo de las expectativas generadas al comienzo de la carrera y la opinión al terminar, obtendremos ciertas concordancias entre los que esperaban que la asignatura fuera muy o relativamente interesante y los que confirmaron dichas expectativas. En el 
antes y el después de la Estadística encontramos un $76,8 \%$ de licenciados que la catalogaban así, elevándose a un $84,1 \%$ para Técnicas de Investigación, aunque en este último caso se advierte una cierta decepción al observarse un desplazamiento de un tercio de los que esperaban que fuera muy interesante a la categoría de «relativamente interesante» al acabar la carrera.

TABLA 8

Opinión sobre la importancia de las asignaturas de Estadística y de Técnicas de investigación social para la profesión de sociólogo/politólogo

\begin{tabular}{lrrlrr}
\hline $\begin{array}{c}\text { Importancia } \\
\text { profesion } \\
\text { Estadística }\end{array}$ & Frecuencia & Porcentaje & $\begin{array}{c}\text { Importancia } \\
\text { profesión } \\
\text { Técnicas }\end{array}$ & Frecuencia & Porcentaje \\
\hline Imprescindible & 25 & 42,4 & Imprescindible & 33 & 51,6 \\
Muy importante & 21 & 35,6 & Muy importante & 21 & 32,8 \\
Poco importante & 11 & 18,6 & Poco importante & 7 & 10,9 \\
Nada importante & 2 & 3,4 & Nada importante & 3 & 4,7 \\
\hline TOTAL & 59 & 100,0 & TOTAL & 64 & 100,0 \\
\hline
\end{tabular}

TABLA 9

Expectativa del interés de las dos asignaturas al comenzar y al finalizar la carrera

\begin{tabular}{|c|c|c|c|c|c|}
\hline $\begin{array}{c}\text { Expectativa } \\
\text { comienzo } \\
\text { Estadística }\end{array}$ & Frecuencia & Porcentaje & $\begin{array}{c}\text { Expectativa } \\
\text { comienzo } \\
\text { Técnicas }\end{array}$ & Frecuencia & Porcentaje \\
\hline No interes. & 7 & 11,7 & No interes. & 7 & 10,8 \\
\hline Rel. interes. & 25 & 41,7 & Rel. interes. & 12 & 18,5 \\
\hline Muy interes. & 28 & 46,7 & Muy interes. & 46 & 70,8 \\
\hline TOTAL & 60 & 100,0 & TOTAL & 65 & 100,0 \\
\hline $\begin{array}{c}\text { Opinión } \\
\text { término } \\
\text { Estadística }\end{array}$ & Frecuencia & Porcentaje & $\begin{array}{l}\text { Opinión } \\
\text { término } \\
\text { Técnicas }\end{array}$ & Frecuencia & Porcentaje \\
\hline No interes. & 7 & 11,7 & No interes. & 7 & 10,8 \\
\hline Rel. interes. & 28 & 46,7 & Rel. interes. & 28 & 43,1 \\
\hline Muy interes. & 25 & 41,7 & Muy interes. & 30 & 46,2 \\
\hline TOTAL & 60 & 100,0 & TOTAL & 65 & 100,0 \\
\hline
\end{tabular}

En cualquier caso, no se discute nunca la importancia de estas materias e, incluso, deberíamos añadir que se pide un refuerz̧o de las mismas, opinión que se hace más evidente al finalizar los estudios. 


\section{Y DESPUÉS, ¿QUÉ?}

Habida cuenta de las reflexiones presentadas en el primer bloque de este artículo acerca del perfil del alumnado de la UNED, creemos que no tiene tanta relevancia hablar de una cuestión en la que se centran muchos de los estudios sobre educación universitaria: el del éxito o el fracaso en la inserción profesional de los licenciados. En nuestro caso, si al comenzar sus estudios un $77 \%$ de los licenciados estaban ocupados, en la actualidad este porcentaje sólo ha sufrido un crecimiento de un diez por ciento que, unido al conocimiento que tenemos sobre la preparación universitaria previa que caracterizaba a nuestros estudiantes, relativiza la trascendencia profesional de los estudios llevados a cabo, desviando la importancia de los mismos al terreno más puramente cultural e intelectual. Este espíritu inquieto se materializa en la demanda de ampliación de conocimientos sobre las materias que nos competen. Y para ello las vías que han seguido con este fin se concentran en la asistencia a cursos de técnicas específicas y en algo tan autónomo como la lectura de bibliografía adecuada.

Sin pretender pecar de insistentes, hemos de concluir reiterando el carácter singular de los licenciados en Ciencias Políticas y Sociología a distancia. 